\title{
Allelic heterogeneity of Proteus syndrome
}

\author{
Anna Buser, ${ }^{1}$ Marjorie J. Lindhurst, ${ }^{1}$ Hannah C. Kondolf, ${ }^{1,2}$ Miranda R. Yourick, ${ }^{1,3}$ \\ Kim M. Keppler-Noreuil, ${ }^{1,4}$ Julie C. Sapp, ${ }^{1}$ and Leslie G. Biesecker ${ }^{1}$ \\ ${ }^{1}$ Medical Genomics and Metabolic Genetics Branch, National Human Genome Research Institute, National \\ Institutes of Health, Bethesda, Maryland 20892, USA
}

Abstract Proteus syndrome is a mosaic disorder that can cause progressive postnatal overgrowth of nearly any organ or tissue. To date, Proteus syndrome has been exclusively associated with the mosaic c.49G > A p.(Glu17Lys) pathogenic variant in AKT1, a variant that is also present in many cancers. Here we describe an individual with severe Proteus syndrome who died at $7.5 \mathrm{yr}$ of age from combined parenchymal and restrictive pulmonary disease. Remarkably, this individual was found to harbor a mosaic c.49_50delinsAG p.(Glu17Arg) variant in $A K T 1$ at a variant allele fraction that ranged from $<0.01$ to 0.46 in fibroblasts established from an overgrown digit. This variant was demonstrated to be constitutively activating by phosphorylation of AKT(S473). These data document allelic heterogeneity for Proteus syndrome. We recommend that individuals with a potential clinical diagnosis of Proteus syndrome who are negative for the p.(Glu17Lys) variant be tested for other variants in AKT1.

Corresponding author: lesb@mail.nih.gov

This is a work of the US government.

Ontology terms: contractures of the large joints; fibrocystic lung disease; increased adipose tissue; lower limb asymmetry; overgrowth; upper limb asymmetry; venous malformation

Published by Cold Spring Harbor Laboratory Press

doi:10.1101/mcs.a005181
[Supplemental material is available for this article.]

\section{INTRODUCTION}

Until this report, a somatic variant in the AKT1 gene-c.49G > A, p.(Glu17Lys)—was the only reported cause of Proteus syndrome (Lindhurst et al. 2011). The AKT1 protein is a serinethreonine kinase that signals in the AKT/PI3K/mTOR pathway. The pathophysiology of Proteus syndrome is attributed to constitutive activation of AKT1 (Carpten et al. 2007). This activation limits apoptosis and promotes growth, among other effects (Carpten et al. 2007). The clinical manifestations are dominated by skeletal overgrowth, but the disorder is highly pleiotropic including central nervous system (CNS) overgrowth and neuronal migration defects, vascular anomalies, overgrowth of many other organs and tissues, and bullous or cystic disease of the lungs. Here we report an individual with severe manifestations of Proteus syndrome who has constitutive AKT activation because of a distinct variant in AKT1.

\section{RESULTS}

Clinical Presentation and Family History

The proband was born by emergent cesarean delivery at 37 wk of gestation to a G1P1 21-yrold mother because of fetal decelerations. He underwent surgical repair of an umbilical

\footnotetext{
${ }^{2}$ Present affiliation: Department of Pathology, Case Western Reserve University, Cleveland, Ohio 44106, USA

${ }^{3}$ Present affiliation: Department of Biology, University of Maryland, College Park, Maryland 20742, USA

${ }^{4}$ Present affiliation: Children's National Medical Center, Washington, D.C. 20010, USA
} 
hernia after birth and was fed via a nasogastric tube because of poor oral intake for the first $13 \mathrm{~d}$. He developed infantile seizures that were treated with an antiepileptic. At birth, he was noted to have slight asymmetric overgrowth of the left leg and arm and vascular markings on both legs, diagnosed as cutis marmorata telangiectasia.

By 4 mo of age, the seizures resolved, and the left leg measured $1.5 \mathrm{~cm}$ longer than the right. Linear verrucous epidermal nevi streaked his left arm, left trunk, and left leg. His left sole had a cobblestone appearance (which we now term "pre- CCTN," the precursor lesion of the cerebriform connective tissue nevus (Keppler-Noreuil et al. 2019)). At 5 mo, a lipoma on the left lower back was noted, and at 7 mo, macrodactyly and divergence of the third and fourth fingers of the left hand were noted. He was hospitalized for pneumonia at 14 mo. At $15 \mathrm{mo}$, his leg length discrepancy had progressed to $5 \mathrm{~cm}$ with a left knee valgus deformity of $30^{\circ}$.

Magnetic resonance imaging at 17 mo showed pleural effusions of the lungs, irregularly shaped vertebral bodies, and an enlarged spleen and portal vein. A CCTN was noted on the left fourth finger and the progressive CCTN on the left sole was associated with deviation of the left great toe into an $\sim 30^{\circ}$ extension at rest. Cutaneous capillary malformations were present on both calves.

At $18 \mathrm{mo}$, he underwent hemiepiphysiostasis surgery with tension band plates placement at the left distal medial femur (Crenshaw et al. 2018). At $21 \mathrm{mo}$, he had a left orchiectomy for removal of a serous borderline cystadenoma, and at $22 \mathrm{mo}$, he had an adenoidectomy. At $2 \mathrm{yr} 2 \mathrm{mo}$, he had a second orthopedic surgery replacing the tension band plates on his left femur and adding additional plates to his left proximal tibia. By 2 yr 5 mo of age, his left knee contracture was $90^{\circ}$, and he had regressed to crawling. Subsequently, an above-the-knee amputation of the left leg was performed. Also, at this time, increased lipomatous lesions on his lower left back, left chest, left abdomen, and bilateral axillae were present. His left foot measured $3 \mathrm{~cm}$ longer than the right, and digits $1-4$ in his left hand were overgrown. He experienced daily emesis, which was attributed to gastroesophageal reflux disease. An esophagoduodenoscopy reported the duodenum was thickened and pale.

At $3.5 \mathrm{yr}$, the left fourth finger was amputated because of progressive bony overgrowth. Respiratory issues starting at $4 \mathrm{yr}$ of age necessitated a chest $\mathrm{CT}$, which showed severe bullous pulmonary disease and cervical vertebrae elongation. He was placed on pediatric hospice care at $6 \mathrm{yr}$. Imaging studies at 6 yr 8 mo showed the bullae and fibrosis had progressed, with an estimate of $40 \%$ normal lung tissue remaining (Fig. 1C). Moreover, severe thoracolumbar scoliosis contributed a restrictive component to his lung disease. Pulmonary function tests showed elevated residual volume (211\%) and reduced total lung capacity (80\%). His oxygen saturations on room air ranged from $70 \%$ to $88 \%$, and, thus, he used supplement oxygen at night and as needed during the day. Further imaging showed a coxa valga deformity in the left femoral head, shortening of the left ulna, and myocardial fat infiltration in his right ventricular septum. A vascular lesion in the left optic nerve region was also reported.

At 6 yr 11 mo, he began taking the AKT inhibitor, miransertib (ARO 092), at a dose of 5 $\mathrm{mg} / \mathrm{m}^{2}$ through a compassionate use protocol. However, because of the patient's worsening condition, the study drug was stopped after 3 mo. The patient died at $7 \mathrm{yr} 6$ mo of age from respiratory complications secondary to advanced bullous lung disease. This individual's manifestations exceeded the 2006 criteria needed to support a clinical diagnosis of Proteus syndrome (Table 1; Biesecker 2006).

\section{Molecular Analyses}

Molecular testing of DNA isolated from five fibroblast cultures from the finger amputation at $3.5 \mathrm{yr}$ using a custom restriction fragment length polymorphism (RFLP) assay (Lindhurst et al. 

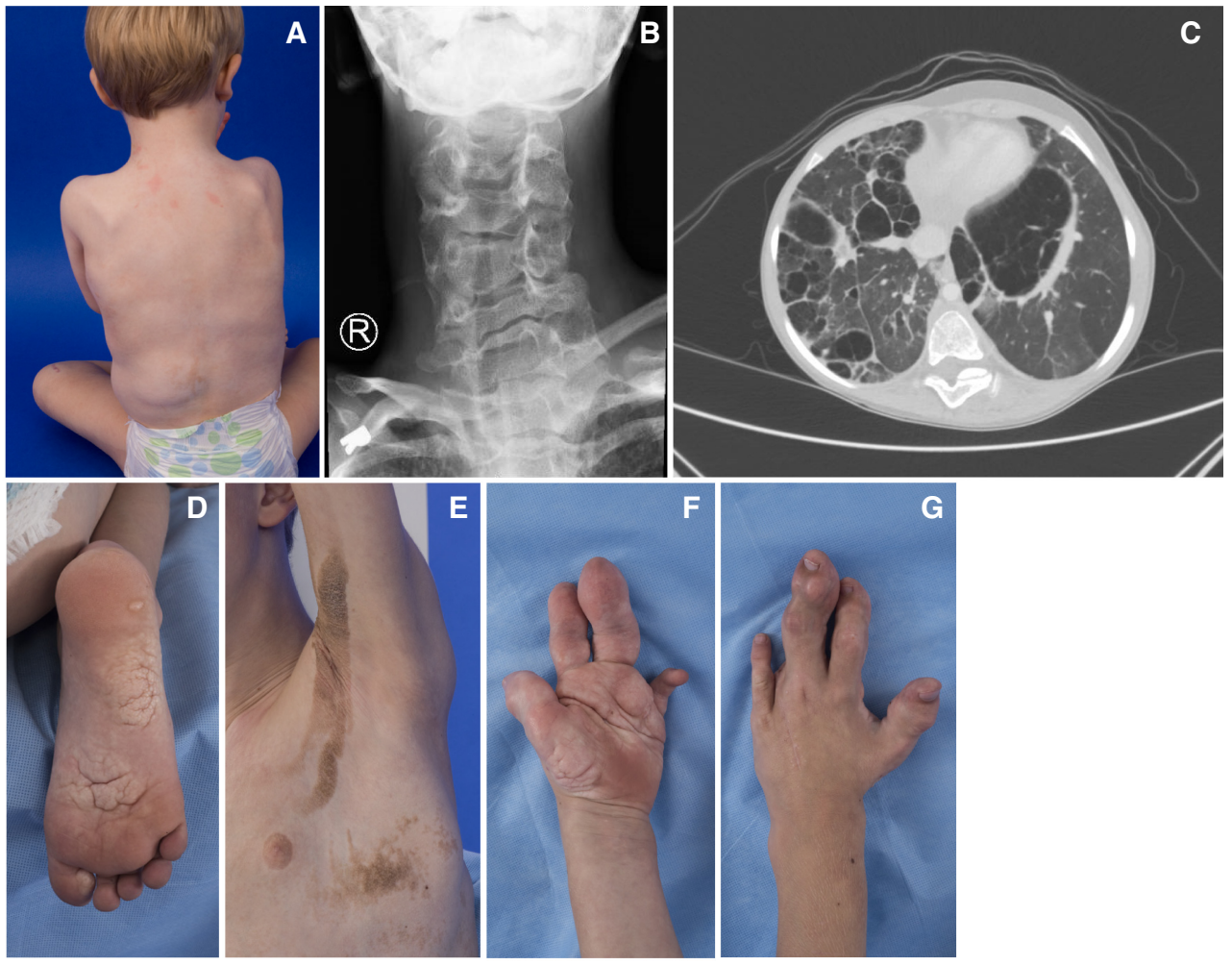

Figure 1. Patient images showing $(A)$ lipoma of left lower back at $22 \mathrm{mo}$; $(B)$ cervical vertebrae elongation and fusion of the posterior elements of $\mathrm{C} 2-\mathrm{C} 3$ and $\mathrm{C} 4-\mathrm{C} 5$ at 6 yr $8 \mathrm{mo} ;(C)$ bilateral pulmonary bullae via chest CT at 6 yr $10 \mathrm{mo} ;(D)$ cerebriform connective tissue nevus (CCTN) of right sole; $(E)$ linear verrucous epidermal nevi streaking upper left arm and trunk; and $(F, G)$ bony and soft tissue overgrowth of first-third digit, post-fourth finger ray amputation. A CCTN is noted on lower palm $(F)$. Images $D-G$ were obtained at 6 yr 8 mo of age.

\begin{tabular}{lc}
\hline Table 1. Proteus syndrome diagnostic criteria in the proband & \\
\hline Proteus syndrome diagnostic criteria $^{\text {a }}$ & Proband \\
\hline Mosaic distribution of lesions & Yes \\
Sporadic occurrence & Yes \\
Progressive course & Yes, severe \\
(A) Cerebriform connective tissue nevus & Yes \\
(B) Linear epidermal nevus & Yes \\
(B) Asymmetric, disproportionate overgrowth & Yes \\
(B) Bilateral ovarian cystadenoma or parotid monomorphic adenoma before second decade & No \\
(C) Dysregulated adipose tissue & Yes \\
(C) Vascular malformations & Yes \\
(C) Bullous lung disease & Yes \\
(C) Facial phenotype & No
\end{tabular}

${ }^{a}$ Diagnosis requires all three general criteria AND either one from (A), two from (B), or three from (C). 
2011) for the expected AKT1 c.49G > A p.(Glu17Lys) variant was negative. Additional testing using custom RFLP assays for the PIK3CA "hotspot" variants (c.3140A > G p.(His1047Arg), c.3140A > T p.(His1047Leu), c.1624G > A p.(Glu542Lys), and c.1633G > A p.(Glu545Lys) (Lindhurst et al. 2012; Keppler-Noreuil et al. 2014) was negative. We next interrogated DNA from two cultures using a custom capture next-generation sequencing (NGS) panel containing $153 \mathrm{PI}$ KK/AKT pathway genes. Examination of the AKT1 sequence in one of the samples (FB1) identified 58/515 (0.113) alternate reads for a c.49_50delinsAG variant. Visualization of the data using the Integrative Genomics Viewer showed that both substitutions always occurred on the same read (Fig. 2A). The second DNA interrogated by custom capture/NGS (FB4) had 3/474 (0.006) alternate reads for the c.49_50delinsAG variant. Sanger sequencing confirmed the presence of both base changes in DNA directly isolated from a CLIA cell line established from the amputated finger (Fig. 2B). The RFLP assay for the

A

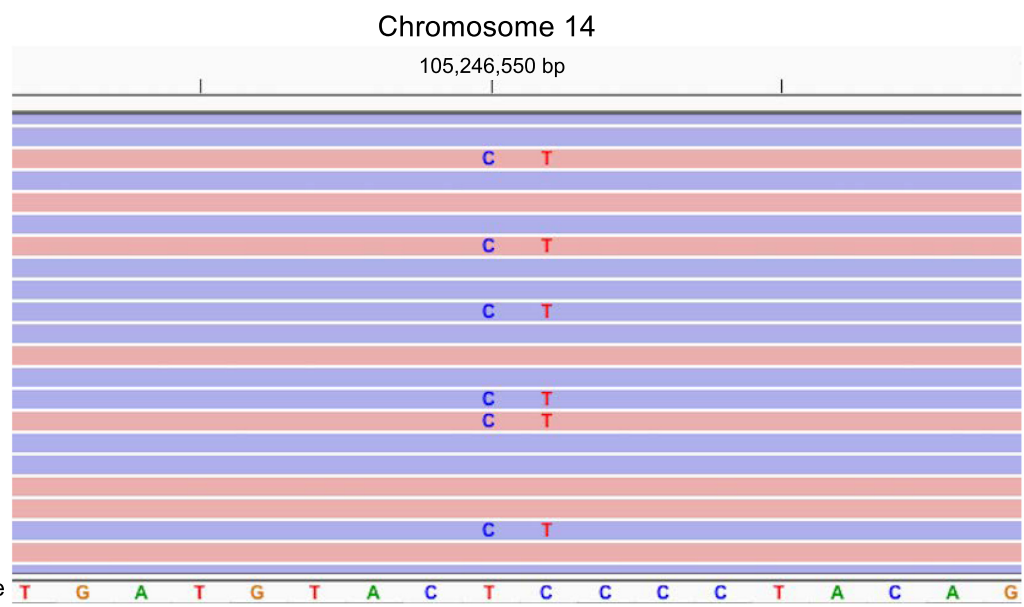

reference base

B

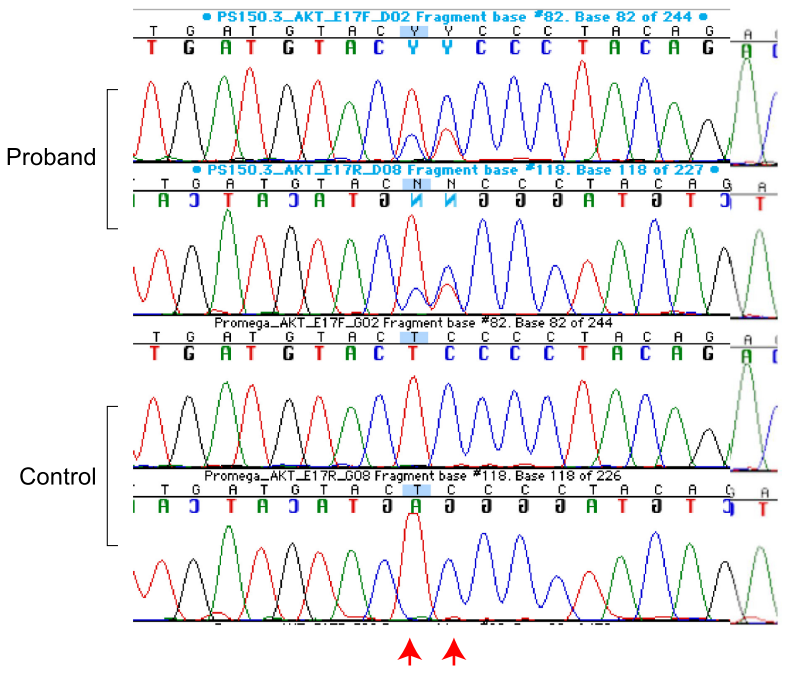

Figure 2. Sequencing results at Chr 14:105,246,550-105,246,551 from the proband. (A) Snapshot of the nextgeneration sequencing reads in sample FB1 visualized using the Integrative Genomics Viewer (Robinson et al. 2011) showed that reads either had both alternate bases or had reference calls at these positions, indicating they were in cis. The color of a row denotes direction of read; note that alternate bases occurred on reads from both directions. (B) Sanger sequencing showed two peaks at each position (arrows) in both the forward and reverse direction that were present in the proband and absent in control DNA. 


\begin{tabular}{lcc}
\hline \multicolumn{3}{l}{ Table 2. Variant levels in fibroblasts from the reported individual } \\
\hline Sample & VAF $\left(\right.$ NGS $\left.^{\mathrm{a}}\right)$ & VAF $\left(\mathrm{RFLP}^{\mathrm{b}}\right)$ \\
\hline FB1 & 0.113 & 0.02 \\
FB2 & NA & 0.46 \\
FB3 & NA & 0.05 \\
FB4 & 0.006 & 0.004 \\
FB5 & NA & 0.31 \\
\hline
\end{tabular}

(VAF) Variant allele fraction, (NGS) next-generation sequencing, (RFLP) restriction fragment length polymorphism.

aFraction of alternate reads at Chr 14:105,246,550-105,246,551 identified by next-generation sequencing.

bariant allele fraction detected by custom restriction fragment length polymorphism assay for the c.50A $>\mathrm{G}$ substitution.

Proteus c.49G > A variant was negative because the additional base change destroys the Mboll restriction site used in the assay. We were unable to develop a RFLP assay that detected the dinucleotide change but instead developed one that detects the c.50A > G substitution component of the 2-bp insertion-deletion (it is not sensitive to the change at nucleotide 49). We tested the fibroblast DNAs and found that all had measurable levels of this change with variant allele fractions (VAFs) ranging from $<0.01$ to 0.46 (Table 2). This further supports that the substitutions are in cis, because only the c.50A $>\mathrm{G}$ and not the c.49G $>A$ assay was positive. The discordance in VAF between the NGS and RFLP results for FB1 was due to the use of DNA isolated from aliquots of the culture that had different admixtures of positive and negative cells. Admixture variation between aliquots is not uncommon for mosaic cell lines (M.J. Lindhurst, unpubl. obs.). We conclude that this individual had a somatic mosaic AKT1 c.49_50delinsAG p.(Glu17Arg) 2-bp variant associated with Proteus syndrome (Table 3).

To test if the p.(Glu17Arg) variant confers constitutive activation, we measured the phosphorylation level of AKT in multiple fibroblast lines from this individual grown in serum-free medium and compared it to levels in control fibroblasts without AKT1 variants and to levels in fibroblast single cell clones from an affected individual with and without the typical p.(Glu17Lys) variant (Fig. 3). Lysates from p.(Glu17Arg)-containing fibroblasts showed a twofold relative activation (FB1 cell line), three- to fourfold relative activation (FB3), a three- to 11-fold (FB4), and a 23- to 25-fold (FB2) increase compared to the averaged levels in the variant-negative lysates. FB2 had a VAF of 0.46 , which is quite similar to the heterozygous affected c.49G > A cloned fibroblasts (VAF 0.5, by definition), the latter showing a 17- to 19-fold increase activity compared to control cells. As expected, these data show a proportionality of VAF to S(473) activation in the various cell lines. We cannot, however, conclude that these levels of VAF or S(473) phosphorylation are relevant to the degree of disease effect in the source tissue as they could instead simply be a local sampling artifact. Given that the $\mathrm{S}(473)$ activation in the VAF $0.46 \mathrm{p}$.(Glu17Arg) sample is as high or higher than that of a cloned p.(Glu17Lys) variant, we conclude that the c.49_50delinsAG p.(Glu17Arg) variant is

\begin{tabular}{|c|c|c|c|c|c|c|c|}
\hline Gene & Chromosome & $\begin{array}{l}\text { HGVS DNA } \\
\text { reference }\end{array}$ & $\begin{array}{l}\text { HGVS protein } \\
\text { reference }\end{array}$ & Variant type & $\begin{array}{l}\text { Predicted } \\
\text { effect }\end{array}$ & $\mathrm{dbSNP/dbVar} \mathrm{ID}$ & Genotype \\
\hline AKT1 & $\begin{array}{l}\text { Chr 14: } \\
\text { g.105,246,550_105,246,551 } \\
\text { delinsCT (GRCh37) }\end{array}$ & c.49_50delinsAG & p.(Glu17Arg) & $\begin{array}{l}\text { Dinucleotide } \\
\text { substitution }\end{array}$ & Missense & VCV000800567.1 & $\begin{array}{c}\text { Mosaic (VAF } \\
0.004-0.46)\end{array}$ \\
\hline
\end{tabular}


COLD SPRING HARBOR Molecular Case Studies
Allelic heterogeneity of Proteus syndrome

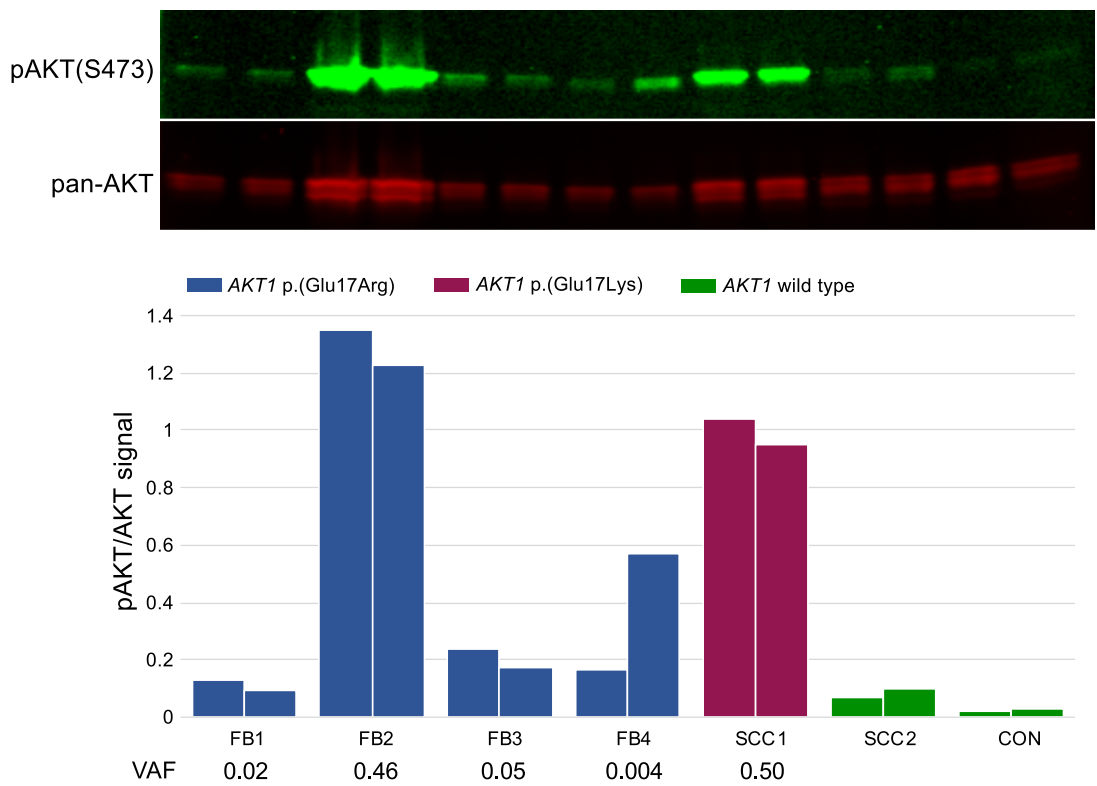

Figure 3. AKT remains active in variant-positive fibroblasts in serum-free medium. Duplicate plates of four fibroblast cultures established from the amputated finger of the proband (blue bars), variant-positive (red bars), and variant-negative single cell fibroblast clones from an individual with a AKT1 c.49G > A; p.Glu17Lys mutation, and fibroblasts from a control individual (green bars) were grown without serum for $24 \mathrm{~h}$ prior to lysis and western analysis. The histogram shows the ratio of the infrared signal from the phospho- and total AKT antibodies. The variant allele fraction (VAF) for each culture is indicated. Cultures with high levels of variant-positive cells (FB2 and SCC1) had high levels of PAKT compared to variant-negative fibroblasts when grown in the absence of growth factors.

at least as strongly activating as, and may have a stronger activating potential than, the more common c.49G > A p.(Glu17Lys) Proteus variant.

\section{DISCUSSION}

Proteus syndrome is a very rare syndrome (prevalence $<1 / 1,000,000$ ) and was first described in the modern medical literature in 1976 (Temtamy and Rogers 1976) and as Proteus syndrome several years later (Wiedemann et al. 1983). The manifestations of Proteus syndrome are associated with an activation of the AKT1 oncogene product (Lindhurst et al. 2011). AKT1 was first recognized to be important for human health when it was found to be mutated in a number of human cancers (COSMIC database; https://cancer.sanger.ac.uk/cosmic) (Tate et al. 2019). Importantly, in most cancers, the variant is the same c.49G > A variant as is found in the mosaic state in individuals with Proteus syndrome. The pathogenesis of Proteus syndrome and the AKT1-mutated cancers is a ligand-independent activation of the AKT1 signal, which is downstream from a number of tyrosine kinase growth factor receptors in the AKT/ PIK3CA/mTOR pathway (Carpten et al. 2007).

Whereas nearly all variants in the COSMIC database are the c.49G > A p.(Glu17Lys) variant, there are a growing number of other activating variants in some less common tumors (Tate et al. 2019). Recent studies have shown heterogeneous AKT1 variants, including inframe duplications in sclerosing pneumocytoma-remarkably, $93 \%$ and $100 \%$ of two cohorts of affected individuals had AKT1 variants (Yeh et al. 2020). An atypical AKT1 p.(Arg23Gln) variant was observed in two neuroendocrine tumors, although causality was not established 
(Park et al. 2019). Finally, one individual with a histopathologically indeterminate epithelioid neoplasm was found to harbor a novel fusion gene comprising the LAMTOR1 and AKT1 genes (Slotkin et al. 2019). From these publications it is clear that allelic heterogeneity is relevant to tumorigenesis and this report is the first to demonstrate this in Proteus syndrome.

This individual unambiguously met the 2006 clinical criteria for Proteus syndrome-he has nearly all manifestations of the disorder (Biesecker 2006). Recently, we have updated these simple clinical criteria to incorporate an integrated clinical-molecular scoring system (Sapp et al. 2019). In this scheme, clinical manifestations are scored on a point scale from -10 to +28 points, with positive points for attributes of Proteus syndrome and negative points for attributes associated with PIK3CA-related overgrowth spectrum. This individual scored 24 points, missing only the criteria for deep vein thrombosis and the facial phenotype. Note that in those updated clinical diagnostic criteria, we specified that diagnosis was based on a dyad of phenotype and genotype and that the latter was a pathogenic variant in AKT1, which we conclude to be the case here. Therefore, we conclude that this individual has AKT1-related Proteus syndrome, even though his variant is different from all other affected individuals to date.

The natural history of the disease in this individual was severe. Although it is known that the mortality of Proteus syndrome is considerable (Sapp et al. 2017), he is one of the younger individuals to expire with this disease. The cause of death was respiratory insufficiency secondary to pulmonary disease, a known manifestation of Proteus syndrome (Lim et al. 2011). The diffuse nature of his bullous disease was such that lobectomy was not felt to be beneficial and he was evaluated as a poor lung transplant candidate because of the advanced nature of the disease and his other medical issues. It is critical to note that his severe parenchymal lung disease was compounded by the restrictive lung disease because of vertebral and chest wall overgrowth and deformation. This combination of respiratory disease is difficult to treat and may primarily be a manifestation that can best be avoided through primary drug treatment (Keppler-Noreuil et al. 2019; Leoni et al. 2019), rather than postsymptomatically.

We conclude that this variant is pathogenic for Proteus syndrome using an adaptation of the ACMG/AMP criteria, which were designed for constitutional variants. We would cite a modification of the PS2 (de novo) criterion, reasoning that a mosaic alteration of this VAF that so strongly matches the phenotype is as strong a piece of evidence as is a constitutional de novo variant. We cite criterion PS3 (functional) in that the AKT phosphorylation assay is a well-established assay that is proximate to the actual pathophysiology of this disease (Carpten et al. 2007). We would cite PM2_Supp (absence in controls) as there is only a single occurrence of this variant in gnomAD, though it is in fact a variant from a tumor sample erroneously included in the database (A O'Donnell, Broad Institute, pers. comm.). We would cite PM5 as codon 17 is known to be mutated in the p.(Glu17Lys) variant of COSMIC and Proteus syndrome (these data could arguably be used instead for the hotspot criterion PM1). We would cite PP2 as multiple in silico programs predict this variant to be deleterious. We would score PP4_Strong as we believe that the phenotype in this patient is highly specific for individuals with variants in this gene; we are aware of no individual with this degree of Proteus syndrome severity and specificity who has a variant in another gene. These total three Strong, one Moderate, and two Supporting, which yields a Pathogenic assertion in (Richards et al. 2015) (their Table 5) and a posterior probability of 0.99993 in the Bayes calculator of (Tavtigian et al. 2018), two orders of magnitude above the Pathogenic threshold.

The detection of a novel variant causing activation of AKT1 in this patient is the first to our knowledge in Proteus syndrome. We have carefully evaluated the COSMIC database and do not see evidence of this variant among the nearly 250 tumors that have AKT1 variants, nor is it reported among the variants detected in sclerosing pneumocytoma (Yeh et al. 2020). We saw no evidence of the c.49_50delinsAG p.(Glu17Arg) variant in COSMIC, although it is possible that that data set may be artifactually depleted for dinucleotide variants for 
methodologic reasons (Tate et al. 2019). We conclude that this variant is a cause of Proteus syndrome, that functional evidence and the severity of the patient could be consistent with a more strongly activating mutational effect (relative to p.Glu17Lys), and that cancer mutational surveys and diagnostic testing for Proteus syndrome should check for other variants in AKT1 if the typical c.49G > A p.(Glu17Lys) variant is not detected.

\section{METHODS}

Fibroblast cultures were established from five separate samples of tissue from the left fourth finger amputation. DNA was isolated using the Gentra Puregene Blood kit (QIAGEN). Genome libraries with 325 base inserts were prepared for each sample from $100 \mathrm{ng}$ of genomic DNA using the Accel-NGS 2S Plus DNA Library Kit (Swift Biosciences). Libraries were barcoded and pooled in an equimolar ratio for capture enrichment. The custom capture panel targeted $0.69 \mathrm{Mb}$ of sequence covering $153 \mathrm{PI}$ 3K/AKT pathway genes. Capture was preformed according to the SeqCap EZ Library SR User's Guide (Roche Nimblegen). The captured exome pool was sequenced on a HiSeq 2500 using version 3 chemistry (Illumina). At least six million paired-end 126 base reads were obtained for each sample. Data were processed using RTA ver. 1.18.64 and CASAVA 1.8.2. The target coverages were calculated using Picard CollectHSMetrics (Picard; http://broadinstitute.github.io/ picard) with default parameters (Base Quality [BO], Mapping Quality $[\mathrm{MO}]=20$ ). The target BED file was converted to the Picard interval file using Picard BedTolntervalList (Supplemental Table S1). The custom RFLP assay used to detect the AKT1 c.50A > G substitution was modified from the c.49G > A assay described in Lindhurst et al. (2011) by using the following primers: (6FAM)-CAGGCATCCCAGGCACATCTGTCC and 5'-AGTAGCGTGGCC GCCAGGTCTTGAGGTAC followed by digestion with Kpnl.

Functional studies were conducted on fibroblasts grown with or without serum for 20$24 \mathrm{~h}$ before cell lysis as described (Lindhurst et al. 2011). Western analyses were performed as described (Lindhurst et al. 2012, 2015) using antibodies to pAKT(S473) and pan-AKT (products \#4060 and \#2920, Cell Signaling Technologies).

\section{ADDITIONAL INFORMATION}

\section{Data Deposition and Access}

The sequencing data is available in the National Center for Biotechnology Information (NCBI) database of Genotypes and Phenotypes (dbGaP; http://www.ncbi.nlm.nih .gov/gap) under the accession number phs002006.v1.p1. The variant has been submitted to ClinVar (https://www.ncbi.nlm.nih.gov/clinvar/) under the accession number

Competing Interest Statement L.G.B. received in-kind research support from ArQule, Inc. and is a Deputy Editor of this Journal.

\section{Referees}

Marcella Zollino

James T. Bennett

Anonymous

Received January 21, 2020; accepted in revised form February 19, 2020.

\section{VCV000800567.1.}

\section{Ethics Statement}

This study was reviewed and approved by the National Human Genome Research Institute (NHGRI) Institutional Review Board, protocol 94-HG-0132. Written informed consent by the mother was provided on behalf of this minor. The mother of the affected individual has reviewed this manuscript and approves publication of the report.

\section{Acknowledgments}

The authors thank the individual's family and the home medical team for their devoted care and advocacy for this young man. The authors thank Celine Hong for completing the dbGap submission and for compiling the sequencing coverage table. The authors thank the 
National Institutes of Health $(\mathrm{NIH})$ Intramural Sequencing Center for performing the NGS capture and sequencing.

\section{Author Contributions}

A.B. and L.G.B. wrote the manuscript; all authors edited it. J.C.S. coordinated and organized clinical data acquisition. K.M.K.-N. clinically evaluated the individual. M.J.L. performed the molecular and functional analyses. H.C.K. and M.R.Y. performed molecular analyses.

\section{Funding}

This study was supported by the intramural research program of the NHGRI, HG200328 and HG200388. Miransertib was provided by ArQule, Inc.

\section{REFERENCES}

Biesecker LG. 2006. The challenges of Proteus syndrome: diagnosis and management. Eur J Hum Genet 14: 1151-1157. doi:10.1038/sj.ejhg.5201638

Carpten JD, Faber AL, Horn C, Donoho GP, Briggs SL, Robbins CM, Hostetter G, Boguslawski S, Moses TY, Savage $S$, et al. 2007. A transforming mutation in the pleckstrin homology domain of AKT1 in cancer. Nature 448: 439-444. doi:10.1038/nature05933

Crenshaw MM, Goerlich CG, Ivey LE, Sapp JC, Keppler-Noreuil KM, Scott AC, Biesecker LG, Tosi LL. 2018. Orthopaedic management of leg-length discrepancy in Proteus syndrome: a case series. J Pediatr Orthop 38: e138-e144. doi:10.1097/BPO.0000000000001121

Keppler-Noreuil KM, Sapp JC, Lindhurst MJ, Parker VE, Blumhorst C, Darling T, Tosi LL, Huson SM, Whitehouse RW, Jakkula E, et al. 2014. Clinical delineation and natural history of the PIK3CA-related overgrowth spectrum. Am J Med Genet A 164A: 1713-1733. doi:10.1002/ajmg.a.36552

Keppler-Noreuil KM, Sapp JC, Lindhurst MJ, Darling TN, Burton-Akright J, Bagheri M, Dombi E, Gruber A Jarosinski PF, Martin S, et al. 2019. Pharmacodynamic study of miransertib in individuals with Proteus syndrome. Am J Hum Genet 104: 484-491. doi:10.1016/j.ajhg.2019.01.015

Leoni C, Gullo G, Resta N, Fagotti A, Onesimo R, Schwartz B, Kazakin J, Abbadessa G, Crown J, Collins CD, et al. 2019. First evidence of a therapeutic effect of miransertib in a teenager with Proteus syndrome and ovarian carcinoma. Am J Med Genet A 179: 1319-1324. doi:10.1002/ajmg.a.61111

Lim GY, Kim OH, Kim HW, Lee KS, Kang KH, Song HR, Cho TJ. 2011. Pulmonary manifestations in Proteus syndrome: pulmonary varicosities and bullous lung disease. Am J Med Genet A 155A: 865-869. doi:10 .1002/ajmg.a.33926

Lindhurst MJ, Sapp JC, Teer JK, Johnston JJ, Finn EM, Peters K, Turner J, Cannons JL, Bick D, Blakemore L, et al. 2011. A mosaic activating mutation in AKT1 associated with the Proteus syndrome. N Engl J Med 365: 611-619. doi:10.1056/NEJMoa1104017

Lindhurst MJ, Parker VE, Payne F, Sapp JC, Rudge S, Harris J, Witkowski AM, Zhang Q, Groeneveld MP, Scott $\mathrm{CE}$, et al. 2012. Mosaic overgrowth with fibroadipose hyperplasia is caused by somatic activating mutations in PIK3CA. Nat Genet 44: 928-933. doi:10.1038/ng.2332

Lindhurst MJ, Yourick MR, Yu Y, Savage RE, Ferrari D, Biesecker LG. 2015. Repression of AKT signaling by ARO 092 in cells and tissues from patients with Proteus syndrome. Sci Rep 5: 17162. doi:10.1038/srep17162

Park HY, Kwon MJ, Kang HS, Kim YJ, Kim NY, Kim MJ, Min KW, Choi KC, Nam ES, Cho SJ, et al. 2019. Targeted next-generation sequencing of well-differentiated rectal, gastric, and appendiceal neuroendocrine tumors to identify potential targets. Hum Pathol 87: 83-94. doi:10.1016/j.humpath.2019.02.007

Richards S, Aziz N, Bale S, Bick D, Das S, Gastier-Foster J, Grody WW, Hegde M, Lyon E, Spector E, et al. 2015. Standards and guidelines for the interpretation of sequence variants: a joint consensus recommendation of the American College of Medical Genetics and Genomics and the Association for Molecular Pathology. Genet Med 17: 405-424. doi:10.1038/gim.2015.30

Robinson JT, Thorvaldsdottir H, Winckler W, Guttman M, Lander ES, Getz G, Mesirov JP. 2011. Integrative genomics viewer. Nat Biotechnol 29: 24-26. doi:10.1038/nbt.1754

Sapp JC, Hu L, Zhao J, Gruber A, Schwartz B, Ferrari D, Biesecker LG. 2017. Quantifying survival in patients with Proteus syndrome. Genet Med 19: 1376-1379. doi:10.1038/gim.2017.65 
Sapp J, Buser A, Burton-Akright J, Keppler-Noreuil K, Biesecker LG. 2019. A dyadic genotype-phenotype approach to diagnostic criteria for Proteus syndrome. Am J Med Genet C Semin Med Genet 181: 565-570. doi:10.1002/ajmg.c.31744

Slotkin EK, Diolaiti D, Shukla NN, Dela Cruz FS, Clark JJ, Gundem G, Yellapantula VD, Levine MF, You D, Ma P, et al. 2019. Patient-driven discovery, therapeutic targeting, and post-clinical validation of a novel AKT1 fusion-driven cancer. Cancer Discov 9: 605-616. doi:10.1158/2159-8290.CD-18-0953

Tate JG, Bamford S, Jubb HC, Sondka Z, Beare DM, Bindal N, Boutselakis H, Cole CG, Creatore C, Dawson E, et al. 2019. COSMIC: the Catalogue Of Somatic Mutations In Cancer. Nucleic Acids Res 47: D941-D947. doi:10.1093/nar/gky1015

Tavtigian SV, Greenblatt MS, Harrison SM, Nussbaum RL, Prabhu SA, Boucher KM, Biesecker LG, ClinGen Sequence Variant Interpretation Working Group. 2018. Modeling the ACMG/AMP variant classification guidelines as a Bayesian classification framework. Genet Med 20: 1054-1060. doi:10.1038/gim.2017.210

Temtamy SA, Rogers JG. 1976. Macrodactyly, hemihypertrophy, and connective tissue nevi: report of a new syndrome and review of the literature. J Pediatr 89: 924-927. doi:10.1016/S0022-3476(76)80597-5

Wiedemann HR, Burgio GR, Aldenhoff P, Kunze J, Kaufmann HJ, Schirg E. 1983. The Proteus syndrome. Partial gigantism of the hands and/or feet, nevi, hemihypertrophy, subcutaneous tumors, macrocephaly or other skull anomalies and possible accelerated growth and visceral affections. Eur J Pediatr 140: 5-12. doi:10 .1007/BF00661895

Yeh YC, Ho HL, Wu YC, Pan CC, Wang YC, Chou TY. 2020. AKT1 internal tandem duplications and point mutations are the genetic hallmarks of sclerosing pneumocytoma. Mod Pathol 33: 391-403. doi:10.1038/ s41379-019-0357-y 


\section{COLD SPRING HARBOR Molecular Case Studies}

\section{Allelic heterogeneity of Proteus syndrome}

Anna Buser, Marjorie J. Lindhurst, Hannah C. Kondolf, et al.

Cold Spring Harb Mol Case Stud 2020, 6: a005181 originally published online April 23, 2020

Access the most recent version at doi: $10.1101 /$ mcs.a005181 Supplementary http://molecularcasestudies.cshlp.org/content/suppl/2020/04/24/mcs.a005181.D
Material

References This article cites 21 articles, 1 of which can be accessed free at: http://molecularcasestudies.cshlp.org/content/6/3/a005181.full.html\#ref-list-1

License This is a work of the US government.

Email Alerting Receive free email alerts when new articles cite this article - sign up in the box at the Service top right corner of the article or click here. 